\title{
Study on control of bird flu outbreak within a poultry farm
}

T. Delia Nova ${ }^{1} \quad$ H. Mawengkang ${ }^{2} \quad$ M. Watanabe ${ }^{3}$

(Received 10 July 2010; revised 8 September 2010)

\begin{abstract}
In a production process of a poultry farm, the entire population of birds is kept constant at the manageable capacity. After intrusion of influenza virus, infected birds remain as a source of infection regardless of being alive or dead, unless they are completely removed. A mathematical model based on these factors is analysed to study time evolution of populations of susceptible birds and infected birds, and concentration of bird flu virus. Numerical results show that the population of domestic birds can be made secure against infection by proper vaccination and proper removal of infected birds.
\end{abstract}

\section{Contents}

\section{Introduction}

C669

2 Modeling bird flu infection in a poultry farm

C670

http://anziamj.austms.org.au/ojs/index.php/ANZIAMJ/article/view/2432 gives this article, (c) Austral. Mathematical Soc. 2010. Published September 23, 2010. ISSN 1446-8735. (Print two pages per sheet of paper.) Copies of this article must not be made otherwise available on the internet; instead link directly to this URL for this article. 
4 Numerical results based on bird flu infection model

\section{Introduction}

Since outbreaks of bird flu (avian influenza) spread widely in 2003, poultry farms have always been under constant threat of loss due to the disease. The disease originates from virulence produced by influenza virus $\mathrm{H} 5 \mathrm{~N} 1$ carried by wild birds. Unlike wild birds, infection of domestic birds causes serious symptoms that eventually lead to death. Primary factors for outbreak of bird flu include existence of avian influenza virus as source of disease, poultry as host, and environment as medium, and are likely to provide opportunities for infection under inappropriate supervision of a poultry farm. Vaccination is an effective measure to reduce the risk of infection both for humans and for domestic animals [1].

A mathematical model consisting of ordinary differential equations has been proposed and analysed to determine time evolution of populations of susceptible birds and infected birds. In a production process on a poultry farm, the entire population of birds is kept constant at the manageable capacity by supply of new healthy birds when vacancies are created, or by shipping of healthy birds when the entire population exceeds the capacity. After intrusion of influenza virus, some of infected birds stay alive and others die. However, infected birds remain as a source of infection regardless of being alive or dead, unless they are completely removed from the entire population. Our mathematical model is based on these factors. Analysis based on the model shows that the population of domestic birds can be made secure against 
infection by proper vaccination and proper removal of infected birds. Analysis also shows that the population cannot be made secure by vaccination alone, and that security can be achieved by removal of infected birds alone without vaccination [5].

The mathematical model is extended to cover time evolution of populations of susceptible birds and infected birds, and concentration of bird flu virus. The model is numerically analysed to simulate evolution of the populations for various parameter values and to propose a control strategy against outbreak of bird flu within a poultry farm.

\section{Modeling bird flu infection in a poultry farm}

Contamination of a poultry farm with bird flu divides the population of domestic birds into two classes: the class of healthy birds susceptible to infection; and the class of infected birds. The si model

$$
\frac{d x}{d t}=c-b x-\omega x y \text { and } \frac{d y}{d t}=\omega x y-(b+m) y,
$$

is analysed to study evolution of the population of susceptible birds $x$ and the population of infected birds $y$ as a part of avian-human influenza model [3]. Here parameter $\mathrm{c}$ is the rate at which new birds are born, parameter $\mathrm{b}$ is the death rate for susceptible birds and infected birds, and parameter $m$ is the additional death rate for infected birds. The term $\omega x y$ denotes the number of susceptible birds infected per unit time, and is proportional to the number of susceptible birds $x$ and the number of infected birds $y$. Equation (1) is called the SI model because S and I stand for susceptible birds and infected birds, respectively, and $x$ and $y$ are the populations of susceptible birds and infected birds, respectively.

The model (1) is not adequate for closed systems such as poultry farms. In 
a poultry production process in a farm, the entire population of domestic birds is balanced with the capacity of the farm by shipping of healthy birds when the entire population exceeds the capacity, and by supply of new birds when vacancies are created by shipping of healthy birds or death of healthy or infected birds. The first two terms on the right hand side of the equation (1) are replaced with $\mathbf{a}\{\mathbf{c}-(\boldsymbol{x}+\mathbf{y})\}$. Parameter $\mathbf{c}$ denotes the capacity of the farm, and parameter a denotes the time rate of supply. All the infected domestic birds eventually die from the disease. Some infected birds die of the disease almost immediately and others stay alive longer, but regardless of being alive or dead, infected birds remain as sources of infection unless they are removed from the population. The rate of removal of infected birds is proportional to the population of infected birds, and so the second term in the right hand side of the equation (1) is replaced with $-m y$, where $m$ is the removal rate. The foregoing discussion leads to the following system of differential equations [5]:

$$
\frac{d x}{d t}=a\{c-(x+y)\}-\omega x y \text { and } \frac{d y}{d t}=\omega x y-m y,
$$

where $\mathbf{c}, \omega$ and $m$ are positive parameters.

Stationary points of system (2) are constant solutions which make the righthand sides equal to zero. The equation $\omega x y-m y=0$ implies that either $x=$ $\mathrm{m} / \boldsymbol{\omega}$ or $y=0$. Then the stationary points of (2) are found by substituting these expressions into $\mathbf{a}\{\mathbf{c}-(\boldsymbol{x}+\mathbf{y})\}-\boldsymbol{w x y}$. One stationary point of the system (2) is

$$
(x, y)=(c, 0),
$$

which corresponds to the state free of infection. Another stationary point of the system (2) is

$$
(x, y)=\left(\frac{m}{\omega}, \frac{a(c \omega-m)}{\omega(a+m)}\right) .
$$

The $\boldsymbol{y}$ component of the stationary point (4) is positive if and only if

$$
c \omega-m>0 .
$$


The stationary point (4) is practically significant under the condition (5), while it is practically insignificant under the condition $c \omega-m<0$. It is desirable that the stationary point (3) is stable in the sense that the state always returns to the original state after change due to intrusion of bird flu. It is shown that stationary point (3) is unstable under the condition (5), and that it is asymptotically stable under the condition $\mathrm{c} \omega-\mathrm{m}<0$. It is also shown that stationary point (4) is asymptotically stable under the condition (5), and that it is unstable under the condition $\mathrm{c} \omega-\mathrm{m}<0$ [5]. The stability of a stationary point $(x, y)=(\xi, \eta)$ of the system (2) depends on the eigenvalues of the Jacobian matrix

$$
A=\left[\begin{array}{cc}
-(a+\omega \eta) & -(a+\omega \xi) \\
\omega \eta & \omega \xi-m
\end{array}\right] .
$$

The stationary point is asymptotically stable when all the eigenvalues of $A$ have negative real parts, and unstable when at least one eigenvalue has a positive real part [2]. Let $\lambda_{-}$and $\lambda_{+}$be the eigenvalues of $A$. Then

$$
\begin{aligned}
& \lambda_{ \pm}=\frac{\operatorname{tr} A}{2} \pm \frac{\sqrt{(\operatorname{tr} A)^{2}-4 \operatorname{det} A}}{2} \\
\text { where } & \operatorname{tr} A=-(a+\omega \eta)+\omega \xi-m, \\
\text { and } & \operatorname{det} A=-(a+\omega \eta)(\omega \xi-m)+\omega \eta(a+\omega \xi) .
\end{aligned}
$$

The steady state solution is asymptotically stable for $\operatorname{tr} A<0$ and $\operatorname{det} A>0$. For the stationary point (3), equations (6)-(8) lead to

$$
\lambda_{-}=-\mathrm{a}, \quad \lambda_{+}=\omega \mathrm{c}-\mathrm{m} .
$$

Under the condition (5), the stationary point (3) is unstable. Under the condition $\mathrm{c} \omega-\mathrm{m}<0$, the stationary point is asymptotically stable.

For the stationary point (4), equations (7) and (8) become

$$
\operatorname{tr} A=-\frac{a(a+c \omega)}{a+m}<0, \quad \operatorname{det} A=a(c \omega-m) .
$$


Under the condition (5), $\operatorname{det} A>0$, and the stationary point (4) is asymptotically stable. It is unstable under the condition $\operatorname{det} A<0$.

In order to propose effective measures against outbreaks of bird flu, it is important to grasp temporal and spatial distribution of virus concentration. However, systems (1) and (2) provide no information concerning virus concentration. Note that the time rate of increase of virus concentration is proportional to itself. Decrease of susceptible birds due to infection is proportional to the population of susceptible birds, and it is also proportional to the virus concentration. Decreased amount of susceptible birds due to infection is the increased amount of infected birds. The rate of increase in virus concentration is controlled by the population of infected birds as hosts. It is positive when the virus concentration falls below the capacity of the hosts, and it becomes negative when the virus population exceeds the capacity of the hosts.

Let $x, y$ and $z$ be the population of susceptible birds, the population of infected birds, and virus concentration, respectively. In production processes of poultry farms, the total population $x+y$ is maintained at the capacity of the farm $c$. Healthy susceptible birds are supplied when vacancies are created $(c-(x+y)>0)$, and the increasing rate due to vacancies is $\mathbf{a}\{c-(x+y)\}$, where $\boldsymbol{a}$ is a positive constant. Susceptible birds becomes infected birds when they are infected. The number of susceptible birds infected per unit time is proportional to the virus concentration in the medium. It is also proportional to the number of susceptible birds. The decreasing rate of susceptible birds due to infection is $\sigma x z$, where $\sigma$ is a positive constant. The time rate of change in the number of susceptible birds is the difference between the increasing rate and the decreasing rate, and

$$
\frac{d x}{d t}=a\{c-(x+y)\}+\sigma x z .
$$

The decreasing rate of the susceptible birds due to infection is the increasing rate of infected birds, and the number of infected birds removed from the entire population is proportional to the number of infected birds, which leads 
to

$$
\frac{d y}{d t}=\sigma x z-m y .
$$

Infected birds are hosts of influenza virus, and the increasing rate of the virus concentration is proportional to the number of infected birds, and decreasing rate of virus is proportional to the virus concentration, which leads to

$$
\frac{d z}{d t}=p y-q z .
$$

Let $r=q / p$ and $\omega=\sigma / r$. The system of equations

$$
\begin{aligned}
& \frac{d x}{d t}=a\{c-(x+y)\}-\omega r x z, \\
& \frac{d y}{d t}=\omega r x z-m y, \\
& \frac{d z}{d t}=p(y-r z) .
\end{aligned}
$$

governs the time evolution of the population of susceptible birds, the population of infected birds, and the virus concentration [6].

\section{Dominant states of bird flu infection model}

Stationary points of the system (9) are constant solutions, obtained by setting the right-hand sides equal to 0 . Let $(\xi, \eta, \zeta)$ be a stationary point of the system (9). The stability of $(\xi, \eta, \zeta)$ is determined by the eigenvalues $\lambda_{1}, \lambda_{2}$ and $\lambda_{3}$ of the Jacobian matrix

$$
A(a, p, r, \omega, \xi, \zeta)=\left[\begin{array}{ccc}
-(a+r \omega \zeta) & -a & -r \omega \xi \\
r \omega \zeta & -m & r \omega \xi \\
0 & p & -p r
\end{array}\right]
$$


The stationary point $(\xi, \eta, \zeta)$ is asymptotically stable when the real parts of $\lambda_{1}, \lambda_{2}$ and $\lambda_{3}$ are all negative [2], and it is unstable when at least one of the eigenvalues has positive real part.

There are two stationary points of system (9). One stationary point is

$$
(x, y, z)=(c, 0,0)
$$

and the other is

$$
(x, y, z)=\left(\frac{m}{\omega}, \frac{a(c \omega-m)}{\omega(a+m)}, \frac{a(c \omega-m)}{r \omega(a+m)}\right) .
$$

Note that the $\boldsymbol{y}$ component and $z$ component of stationary point (11) are positive, and that the stationary point is practically significant if and only if the condition (5) holds. For $\mathrm{m}=0$, stationary point (11) is

$$
(x, y, z)=\left(0, c, \frac{c}{r}\right)
$$

which coincides with the stationary point (10) for $m=c \omega$. As $m$ increases from 0 to $\mathrm{c} \omega$, stationary point (11) moves on a curve connecting the points (12) and (10).

For the stationary point (10), the eigenvalues of the matrix $A$ are

$$
\begin{aligned}
& \lambda_{1}=-a \\
& \lambda_{2}=-\frac{m+p r}{2}-\frac{\sqrt{(m-p r)^{2}+4 p r \omega c}}{2} \\
& \lambda_{3}=-\frac{m+p r}{2}+\frac{\sqrt{(m-p r)^{2}+4 p r \omega c}}{2}
\end{aligned}
$$

All the eigenvalues are real. For $0 \leqslant m<\omega c, \lambda_{1}$ and $\lambda_{2}$ are negative, but $\lambda_{3}$ is positive, and the stationary point (10) is unstable. For $m>\omega c$, all the eigenvalues are negative and the stationary point (10) is asymptotically stable. 
The eigenvalues of $A=A(a, p, r, \omega, \xi, \zeta)$ associated with the stationary point (11) have been evaluated for the special case $a=p r$. In this case, the eigenvalues $\lambda_{1}, \lambda_{2}$ and $\lambda_{3}$ of $A$ are

$$
\lambda_{1}=-a, \quad \lambda_{2}=-(a+m), \quad \lambda_{3}=-\frac{a}{a+m}(c \omega-m) .
$$

For $0 \leqslant m<\omega c$, all the eigenvalues are negative and the stationary point (11) is asymptotically stable. For $m>\omega c, \lambda_{1}$ and $\lambda_{2}$ are negative, but $\lambda_{3}$ is positive, and the stationary point (11) is unstable.

The results concerning the stability of the stationary points indicate that the stationary point (11) is asymptotically stable when it is practically significant $(\mathrm{c} \omega-\mathrm{m}>0)$, and that the stationary point (10) becomes asymptotically unstable when the stationary point (11) becomes practically insignificant $(\mathrm{c} \omega-\mathrm{m}<0)$.

\section{Numerical results based on bird flu infection model}

System (9) was solved numerically for 100 initial values

$$
x=i \times 0.5, \quad y=j \times 0.5, \quad z=k \times 0.5,
$$

for $i, j, k=0,1,2,3$ and 4 using the fourth order Adams-Bashforth-Moulton predictor-corrector in PECE mode in conjunction with the Runge-Kutta Method to generate values of approximate solution at the first three steps [4]. Here numerical results were obtained for $m=l \times 0.25, l=0,1, \ldots, 20$ [6], and with time step 0.001 for 2,000,000 steps. Figures 1-3 show the stationary points (10) and (11), and the numerical solutions for $a=1, c=1, \omega=2$, $\mathrm{p}=1$, and $\mathrm{q}=1$, and for three different values of $\mathrm{m}: \mathrm{m}=1.75, \mathrm{~m}=2.0$ and $m=2.25$. For $0 \leqslant m<2.0$, all the numerical solutions terminated in the sphere of radius $10^{-6}$ centered at the stationary point (11), which confirms 


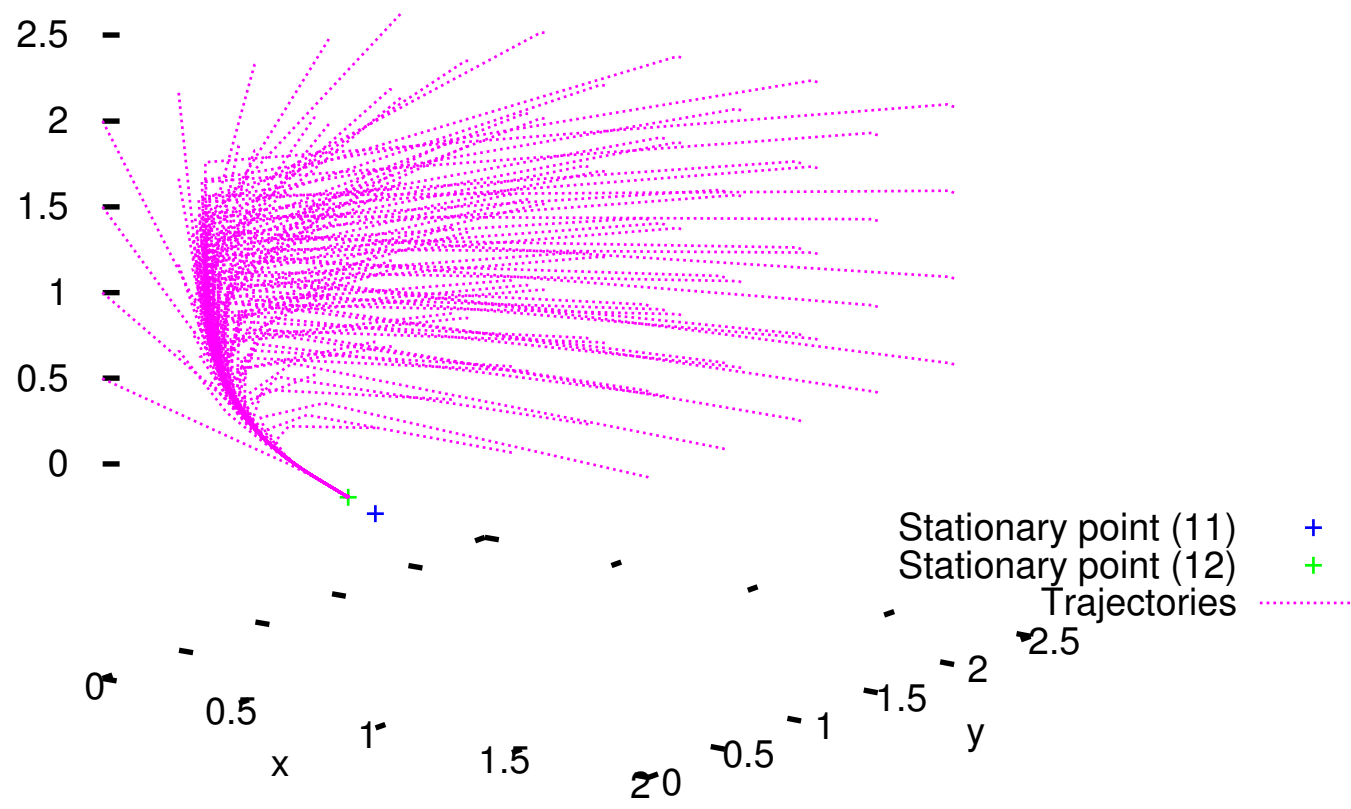

Figure 1: Stationary points and trajectories for $a=1, c=1, \omega=2$, $\mathrm{m}=1.75, \mathrm{p}=1, \mathrm{q}=1$.

that the stationary point (11) is asymptotically stable for those values of $\mathrm{m}$. For $2.0<m \leqslant 5.0$, all the numerical solutions terminated in the sphere of radius $10^{-6}$ centered at the stationary point (10), which confirms that the stationary point (10) is asymptotically stable for those values of $\mathrm{m}$. 


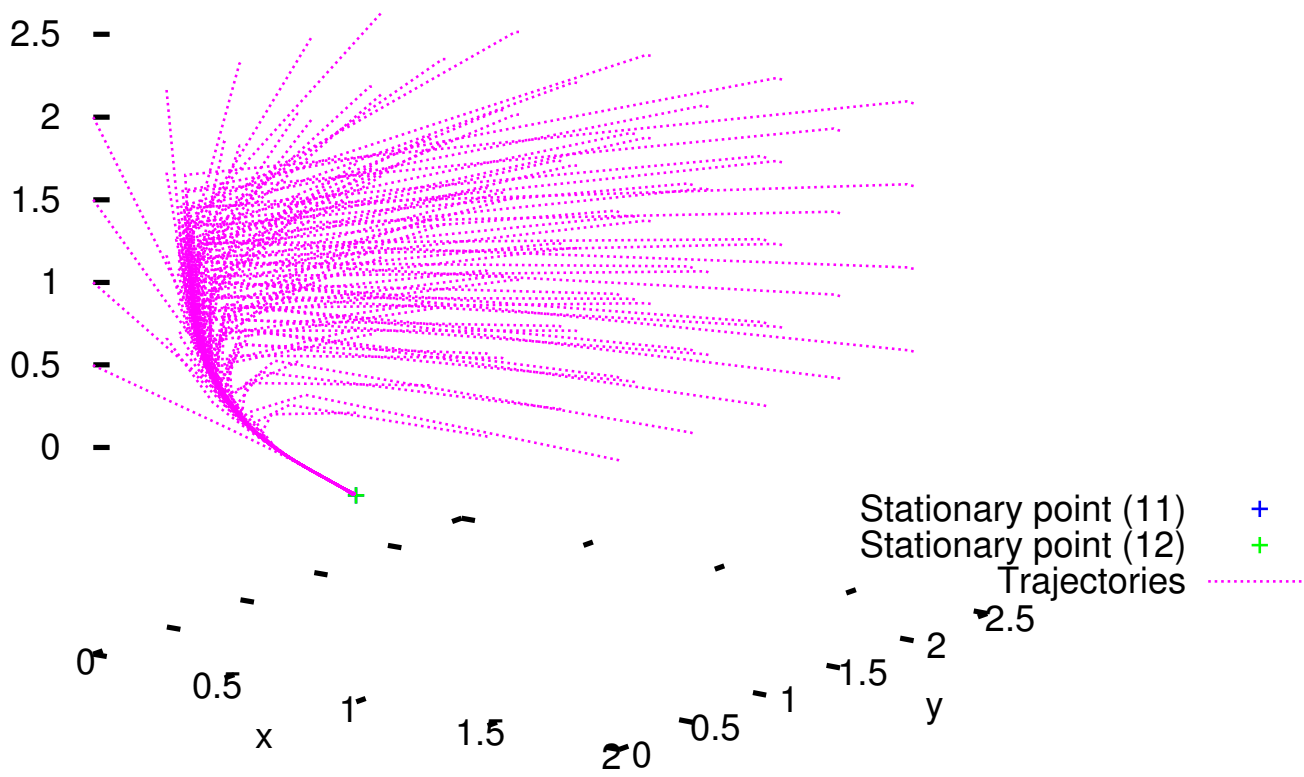

Figure 2: Stationary points and trajectories for $a=1, c=1, \omega=2$, $\mathrm{m}=2.0, \mathrm{p}=1, \mathrm{q}=1$. 
Z

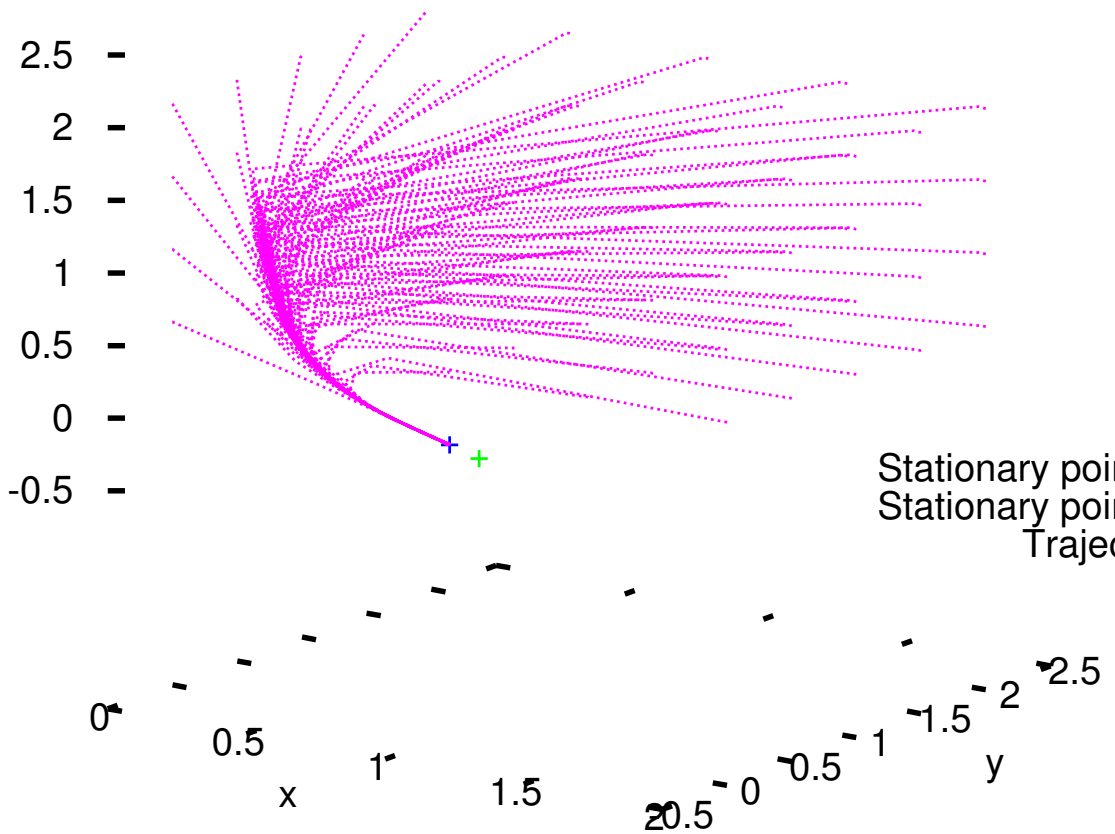

FiguRE 3: Stationary points and trajectories for $\mathrm{a}=1, \mathrm{c}=1, \omega=2$, $\mathrm{m}=2.25, \mathrm{p}=1, \mathrm{q}=1$. 


\section{Discussion}

Recall that $m$ denotes the removal rate of infected birds, and the state of no infection $(x, y, z)=(c, 0,0)$ is asymptotically stable for all sufficiently large values of $\mathbf{m}$. Our analysis based on the model (9) shows that outbreak can be prevented by proper removal of infected birds, and removal of infected birds is essential for prevention of outbreak within a poultry farm as is shown in analysis of the previous model.

In practice, spot-checks are conducted to detect infection by the $\mathrm{H} 5 \mathrm{~N} 1$ virus. Some birds are taken randomly from a flock, and if one bird is found positive for infection, all the birds in the farm are disposed. This current management practice is not consistent with our model.

In order to make our proposed management procedure practicable, it is necessary to develop a detection system to cover the entire population in an appropriate time span for proper removal of infected birds. If that can be achieved, our results show that it will only be necessary to dispose of infected birds, not all the birds in the farm.

Acknowledgements This work was supported by JSPS KAKENHI 20540118.

\section{References}

[1] Johann Breytenbach. Vaccination and biosecurity is the key. ProQuest Agriculture Journals Poultry World, 33(4):159, Apr 2005. C669

[2] Earl A. Coddington and Norman Levinson. Theory of Ordinary Differential Equations. Robert E. Krieger Publishing Company, Malabar, Florida, 1984. (Original Edition 1955, McGraw Hill Co., Inc.). C672, C675 
[3] Shingo Iwami, Yasuhiro Takeuchi, and Xianning Liu. Avian-human influenza epidemic model. Mathematical Biosciences, 207. C670

[4] J. D. Lambert. Computational Methods in Ordinary Differential Equations. John Wiley \& Sons, Chichester, 1973. C676

[5] Tertia Delia Nova, Herman Mawengkang, and Masaji Watanabe. Modeling and analysis of bird flu outbreak within a poultry farm. In Proceedings of the First International Conference on Bioinformatics, page 96, 2010. C670, C671, C672

[6] Tertia Delia Nova, Herman Mawengkang, and Masaji Watanabe. Study on infection process of bird flu within poultry farm based on modeling and simulation. In Proceedings, International Conference on Environmental Research and Technology (ICERT 2010), 2-4 June 2010, Parkroyal Penang, Malaysia, page 435, 2010. C674, C676

\section{Author addresses}

1. T. Delia Nova, Faculty of Animal Husbandry, Andalas University, West Sumatera, IndONESIA. mailto:delianovatertia@yahoo.com

2. H. Mawengkang, Graduate School of Natural Resources and Environmental Management, University of Sumatera Utara, Medan, INDONESIA. mailto: hmawengkang@yahoo.com

3. M. Watanabe, Graduate School of Environmental Science, Okayama University, Okayama, JAPAN. mailto:watanabe@ems.okayama-u.ac.jp 